\title{
ECMO in Emergency Medicine
}

\author{
Yan Wang* \\ Department of Emergency Medicine, Institute for Environmental Medicine and the Center of Lung Biology \\ University of Pennslyvania, USA
}

Submission: September 01, 2016 ; Published: September 13, 2016

*Corresponding author: Yan Wang, Department of Emergency Medicine, Institute for Environmental Medicine and the Center of Lung Biology ,University of Pennslyvania, 510 21st Street NW Unit 317 Washington DC 20006, USA

\begin{abstract}
ECMO is the extracorporeal membrane oxygenation in the aim to provide extracorporeal life support in both cardiac and respiratory function, it is an important therapy in Cardiacvascular Medicine and Emergency Medicine. When patients unable to keep organ basic required oxygen or blood exchange, ECMO is used to remove patient's blood into artificial tube system on oxygenation and detoxication exchange. ECMO can apply to children and adult for a life-sustain therapy, and it is the choice to allow time for intrinsic recovery of heart and lung from varied critical diseases.
\end{abstract}

Abbreviations: ECMO: Extracorporeal Membrane Oxygenation; ICU: Intensive Care Unit; VA: Veno-Arterial; VV: Veno-Venous

\section{Introduction}

ECMO is the extracorporeal membrane oxygenation in the aim to provide extracorporeal life support in both cardiac and respiratory function; it is an important therapy in Cardiovascular Medicine and Emergency Medicine. When patients unable to keep organ basic required oxygen or blood exchange, ECMO is used to remove patient's blood into artificial tube system on oxygenation and detoxication exchange. ECMO can apply to children and adult for a life-sustain therapy, and it is the choice to allow time for intrinsic recovery of heart and lung from varied critical diseases. From the first ten years development since 1953, ECMO has experienced the initial time to create the ECMO system in Medicine, in 1953, Gibbon used the artificial oxygenation support system on the first case of open heart operation and got successful; and In 1965, Rashkind's were being the first to use the support system in neonatal respiratory failure, till now, ECMO has became the basic therapy required in Cardiovascular or Surgery Department and Intensive Care Unit(ICU) [1].

ECMO is the machine with two major types as the venoarterial (VA) and veno-venous (VV) ECMO. For the two types, blood is commonly drained original from the venous system to the outside of body, then in VA ECMO, it standards use cardiopulmonary bypass through transthoracic cannulation under general anesthesia and make oxygenated blood returned to body circulation system, and in VV ECMO, the blood is returned to the venous system only. VA ECMO helps support the cardiac output, it provides treatment for cardiac or respiratory failure; VV ECMO is only for respiratory failure.

ECMO is effective therapy at varied emergency situation, the indication for children application follows the diagnosis and overcoming on diseases of neonatal respiratory distress syndrome, asphyxia, cardiac shock, surgery or congenital diaphragmatic hernia; also, the application is required to meet the selection criteria in ventilation at maximal ventilatory support is getting to $100 \% \mathrm{O}_{2}$, fraction of inspired oxygen equals to 1 and the peak aspiratory pressures is often as high as $35 \mathrm{~cm}$ water; however, the failure to meet the selection criteria is a relative contraindication for ECMO [1,2]. For ECMO application in adult, it apples in adult respiratory distress syndrome, cadiac surgery, emergency treatment, organ transplantation or others $[3,4]$. As a case observation ECMO can defend some critical ill as acute fulminant myocarditis with cardiogenic shock, the report date presented that VA-ECMO offered an overall survival in patients suffering from myocarditis-induced cardiogenic shock comprised between 55\% and 78\% [5], today less strict selection criteria on patients in certain institutions have made ECMO to more diverse application.

ECMO can use for shorter or longer term life sustaining for over 10 days, and it's complication are those associated with cannulation, vascular disruptions, emboli, infection or those with systemic anticoagulation reaction as gastrointestinal bleeding or intracranial bleeding. VA ECMO has higher risk of complication 
than VV ECMO. I have worked as being Emergency Doctor in Shanghai Children's hospitals previously, ECMO is applied in ICU and cardiac surgery, the experience is shown ECMO is a valuable to therapy in clinical for varied critical situation. Conclusion of the development history, references report and experience, the future for ECMO is seeking more effective therapy by programs on clinical observation, skill education or complication defend on life sustain in order to have more time to get recovery from diseases [2,5].

\section{References}

1. Wang Y (1996) Extracorporeal membrane oxygenation in Clinical Pediatric application. Journal of Pediatric Emergency Medicine 3 (2): 84-85.
2. Carey WA, Colby CE (2009) Extracorporeal membrane oxygenation the treatment of neonatal respiratory failure. Semin Cardiothorac Vasc Anesth 13(3): 192-197.

3. Fiser WP, Yetman AT, Gunselman RJ, Fasules JW, Baker LL, et al. (2003) Pediatrics arteriovenous extracorporeal membrane oxygenation (ECMO) as a bridge to cardiac transplantation. J Heart Lung Transplant 22(7): 770-777.

4. Kulkarni T, Sharma NS, Diaz-Guzman E (2016) Extracorporeal membrane oxygenation in adults: A practical guide for internists. Cleve Clin J Med 83(5): 373-84.

5. Pozzi M, Banfi C, Grinberg D, Koffel C, Bendjelid K, et al. (2016) Venoarterial extracorporeal membrane oxygenation for cardiogenic shock due to myocarditis in adult patients. J Thorac Dis 8(7): 495-502. 